\title{
Modeling of single-molecule FRET-experiments on protein folding: from coarse-grained to all-atom simulations
}

\author{
Vladimir A. Andryushchenko \\ IT SB RAS, Novosibirsk, Russia \\ NSU, Novosibirsk, Russia \\ vladimir.andryushchenko@gmail.com
}

\author{
Sergei F. Chekmarev \\ IT SB RAS, Novosibirsk, Russia \\ NSU, Novosibirsk, Russia \\ chekmarev@itp.nsc.ru
}

\begin{abstract}
A key question in the application of the singlemolecule Förster resonance energy transfer (smFRET) to study protein folding is how the dyes can affect the process of folding. Understanding of these effects is particularly important for small proteins, for which the dyes can be comparable in size with the protein. Also, the smFRET-experiments require a support from simulations in order to interpret the results of the measurements.
\end{abstract}

Keywords - FRET-experiments protein folding molecular dynamics

\section{Motivation}

A key question in the application of the single-molecule Förster resonance energy transfer (smFRET) to study protein folding is how the dyes can affect the process of folding. Understanding of these effects is particularly important for small proteins, for which the dyes can be comparable in size with the protein. Also, the smFRET-experiments require a support from simulations in order to interpret the results of the measurements.

\section{Results}

In this work, we modeled smFRET-experiments on folding of BBL domain, the protein that consists of 45 amino acid residues $[1,2]$. The FRET protein construct is a system that is presented by the protein and two dyes at the protein termini, as it is in the experiment.

First, to gain a general insight into the problem, a coarsegrained representation of the FRET-construct was used [3]. Specifically, the amino acid residues were replaced with monomers at the positions of $\mathrm{C}$-alpha atoms, and the dyes were represented by chains of monomers mimicking the fluorophores and linkers. Using the method of molecular dynamics (MD), the simulation of the folding of the protein itself (as a "baseline" system) and of its FRET-construct was performed. It was found that the presence of dyes and, in particular, the appearance of the excluded volume in the spatial protein conformations due to the dyes, does not prevent the protein from folding into its native state. Also, due to the loading of the FRET-constructs with massive dyes, the FRETconstruct folds considerably slower than the protein itself, which leads to a significant increase in the average folding time.

This preliminary study was followed by the most realistic MD simulation available up-to-date - the protein and dyes were described at atomic level of resolution, an explicit solvent was used, and a brute-force modeling of folding trajectories from an extended state of the protein was performed. As the dyes in the FRET-construct, the Cy3 and Cy5 fluorophores were used, which were attached to the protein termini. The protein and 25,000 water molecules (TIP3P) modeling the solvent were placed in a cube with a 95 A edge and periodic boundary conditions. The simulations were performed by the MD method in the framework of the CHARMM36m program [4]. It was found that at $\mathrm{T}=300 \mathrm{~K}$, the native states of the protein and the FRET-construct are stable. Fifteen MD folding trajectories for both the protein and FRET-construct were generated. It has been found that the presence of dyes does not change the overall picture of folding except that the FRET-construct folds considerably slower than the original protein. The FRET-efficiency histograms constructed on the basis of simulated MD-trajectories have a tendency to converge and are found in agreement the experimental histograms.

\section{ACKNOWLEDGMENT}

This work was supported by a grant from the Russian Foundation for Basic Research (18-04-00013).

\section{REFERENCES}

[1] F. Huang, L. Ying, A.R. Fersht, Direct observation of barrier-limited folding of BBL by single-molecule fluorescence resonance energy transfer, Proc. Natl. Acad. Sci. U. S. A. 106 (2009) 16239-16244.

[2] J. Liu, L.A. Campos, M. Cerminara, X. Wang, R. Ramanathan, D.S. English, V. Muñoz, Exploring one-state downhill protein folding in single molecules, Proc. Natl. Acad. Sci. U. S. A. 109 (2012) 179-184.

[3] S. F. Chekmarev. How the dyes affect folding of small proteins in single-molecule FRET experiments: A simulation study. Biophys. Chem. 254 (2019) 106243.

[4] Brooks BR, Brooks CL III, Mackerell AD Jr, Nilsson L, Petrella RJ, Roux B, et al. CHARMM: the biomolecular simulation program. J. Comput. Chem. 30 (2009)1545-1614. 